\title{
SYNTHESIS AND CHARACTERIZATION OF NOVEL ORGANO-INORGANIC HYBRID MATERIAL OF POLY(3,4-ETHYLENE DIOXYTHIOPHENE) AND PHOSPHOMOLYBDATE ANION
}

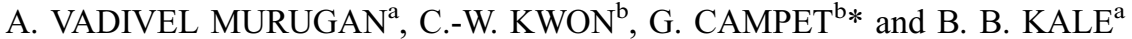 \\ ${ }^{a}$ Centre for Materials for Electronics Technology, Ministry of Communication and Information \\ Technology, Govt. of India, Panchawati, Off-Pashan Road, Pune-411008, India; ' Institut de Chimie \\ de la Matière Condensée, Condensée de Bordeaux du CNRS, 87 Avenue du Dr. A. Schweitzer, \\ 33608 Pessac Cedex, France
}

(Received 31 August 2002; In final form 17 November 2002)

\begin{abstract}
The organo-inorganic hybrid material, consisting of Poly(3,4-Ethylene Dioxythiophene) (PEDOT) doped with phosphomolybdate cluster anions $\left[\mathrm{PMo}_{12} \mathrm{O}_{40}\right]^{3-}$, has been synthesized by direct in situ oxidative polymerization of 3,4-Ethylene Dioxythiophene (EDOT) with phosphomolybdic acid $\left(\mathrm{H}_{3} \mathrm{PMo}_{12} \mathrm{O}_{40}\right)$. Its characterization is investigated by Fourier Transform Infrared Spectroscopy (FT-IR) and Scanning Electron Microscopy (SEM). The hybrid material presents predominantly high electronic conductivities of around 2.0 and $7.0 \mathrm{~S} \mathrm{~cm}^{-1}$ at 300 and $400 \mathrm{~K}$ respectively.
\end{abstract}

Keywords: 3,4-Ethylene Dioxythiophene; Phosphomolybdic acid; Organo-inorganic hybrid material

\section{INTRODUCTION}

The enormous interest demonstrated in the last few years for the research in electronically conducting polymer based on organo-inorganic hybrid material is mainly due to the large number of potential technological applications such as rechargeable lithium batteries, super capacitors, electro-optical displays, shielding for electromagnetic radiations, antistatic packages and conductive coatings [1-3]. In addition, some transition metals in their highest oxidation state can form metal-oxygen cluster anions, usually known as polyoxometalates. They have been extensively studied from a molecular and structural point of view and for their significance in different fields such as electrochemistry, materials science, and photocatalysis either as homogeneous catalysis or supported onto polymers [4-7]. More significantly, the doping of polymers with highly electroactive polyoxometalates in order to achieve high $p$-type polaronic conductivity is a very exciting subject. Related to that, Gomez-Romero, Otero and their co-workers have successfully incorporated inorganic cluster anions into polymer matrix [7, 8]. Among the most conducting polymers, Poly(3,4-Ethylene Dioxythiophene)

* Corresponding author.

ISSN 0882-7516 print; ISSN 1563-5031 online (C) 2003 Taylor \& Francis Ltd

DOI: $10.1080 / 0882751031000073896$ 
(PEDOT) is the most attractive one as it has been reported to exhibit greatly enhanced stability and to be more "environment friendly" compared to polypyrrole and polyaniline [10]. Therefore, it has been attracting growing interest for applications in supercapacitors and lithium ion batteries [11]. Our recent work demonstrated the successful inclusion of PEDOT within the interlamellar gap of the $\mathrm{V}_{2} \mathrm{O}_{5}$ host material, leading to enhanced conductivity and bi-dimensionality; therefore, the resulting hybrid material is a highly promising cathode material for rechargeable lithium batteries $[12,13]$. In this context our aim was to incorporate the following $\left[\mathrm{PMo}_{12} \mathrm{O}_{40}\right]^{3-}$ polyoxometallate, which is highly electroactive, into a PEDOT polymer matrix, with the specific purpose of studying the corresponding hybrid material in its novel function as a conductive insertion electrode for lithium batteries.

For sake of clarity, in the present paper we will solely report the synthesis and characterization of this hybrid molecular material.

\section{EXPERIMENTAL}

3,4-Ethylene Dioxythiophene (EDOT) purchased from Bayer AG (Germany) and phosphomolybdatic acid $\left(\mathrm{H}_{3} \mathrm{PMo}_{12} \mathrm{O}_{40}\right)_{x} \mathrm{H}_{2} \mathrm{O}$ from Loba Chemi (India) were used after further purification. All experiments were conducted with double distilled water. In order to synthesize the hybrid material, $1 \mathrm{ml}$ of pure EDOT was first added to $5 \mathrm{~g}$ of solid phosphomolybdic acid $\left(\mathrm{H}_{3} \mathrm{PMo}_{12} \mathrm{O}_{40}\right)$. Then, the two reagents were intimately mixed by vigorous magnetic stirring. The reaction was evidenced by the formation of a paste whose bluish black color accounts for the reduction of the anion concomitant with the oxidation of EDOT. $50 \mathrm{ml}$ of water was added afterwards, and the mixture was bubbled with oxygen for at least $28 \mathrm{~h}$ with magnetic stirring. Finally a bluish black fine precipitate of phosphomolybdate doped polymer hybrid [PEDOT/ $\mathrm{PMo}_{12}$ ] was obtained, filtered, and dried under dynamic vacuum. Fourier transformed infrared spectroscopy spectra were recorded from pressed $\mathrm{KBr}$ pellets using a Perkin-Elmer Spectrum-2000 FT-IR Spectrometer. Electronic conductivity measurements were made, by using a four probe conductivity method, on samples in pellet form ( $1 \mathrm{~cm}$ diameter, $0.1 \mathrm{~cm}$ thickness) compacted at $5 \mathrm{t} / \mathrm{cm}^{2}$. Scanning electron microscopy (SEM) images were taken on a Philips XL-30 microscope after mounting samples on Al stubs with gold coatings.

\section{RESULTS AND DISCUSSION}

Figures 1(a) and 1(b) show the FTIR spectra of the phosphomolybdic acid and of the chemically synthesized PEDOT/PMo 12 hybrid material respectively. The assignment of the bands is reported in Table I. The bands in the range $787-1100 \mathrm{~cm}^{-1}$, observed in Figure 1(a), correspond mainly to $\mathrm{PMo}_{12}$ [15]. Figure 1(b) shows the bands in the region $1100-1470 \mathrm{~cm}^{-1}$ characteristic of the PEDOT polymer; the asterisks mark the peaks assigned to $\mathrm{PMo}_{12}$. There are clearly significant changes for some bands of the $\mathrm{PMo}_{12}$ anion anchored in the polymer matrix compared to those of the free acid (Table I). The main changes occur for the Mo-O-Mo vibrations, which involve the basic oxygens concerned in the protonation of the anions. The characteristic $\mathrm{Mo}-\mathrm{O}-\mathrm{Mo}$ (corner shared) band shifts to lower frequencies in the hybrid whereas the $\mathrm{Mo}-\mathrm{O}-\mathrm{Mo}$ (edge shared) band shifts to higher frequencies. This points to a strong change in the environment of the $\mathrm{PMo}_{12}$ anion dispersed in the polymer matrix, where it would be surrounded by the more polarizable organic polymer cations. 


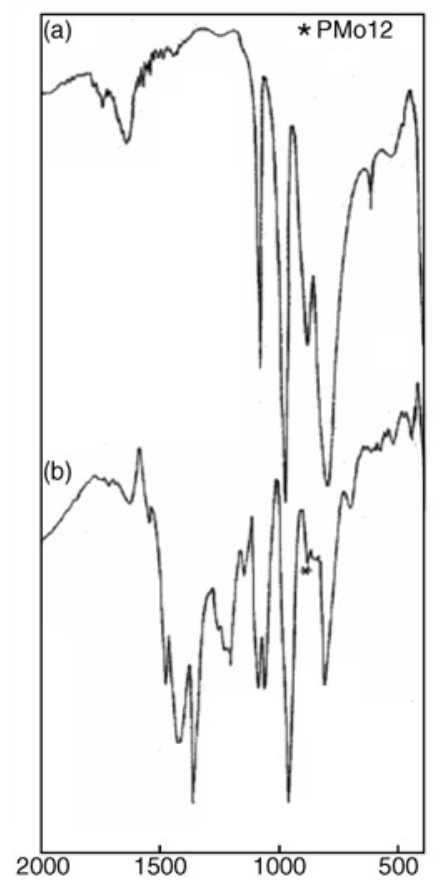

FIGURE 1 FTIR spectra of (a) phosphomolybdic acid and (b) PEDOT/PMo ${ }_{12}$ hybrid material. Asterisks mark the peaks assigned to the $\left[\mathrm{PMo}_{12} \mathrm{O}_{40}\right]^{3-}$. Frequency values reported in abscissa axis are in $\mathrm{cm}^{-1}$.

The chemical composition of the chemically synthesized PEDOT/PMo 12 hybrid was determined using X-ray energy dispersion analysis (EDAX). It reveals a ratio of nine EDOT rings per $\mathrm{PMo}_{12}$ molecule in agreement with the expected value for a fully oxidized polymer with anion $\left[\mathrm{PMo}_{12} \mathrm{O}_{40}\right]^{3-}$ balancing the charges (Scheme 1). In principle, one could suppose that the anion $\left[\mathrm{PMo}_{12} \mathrm{O}_{40}\right]^{3-}$ is reduced to a more negatively charged species (Mo being partially reduced from $6^{+}$to $5^{+}$state) upon oxidation of EDOT to PEDOT. However, the reduction of the $\mathrm{PMo}_{12}$ anion can take place with a simultaneous protonation process that would maintain the overall $3^{-}$charge $[16,17]$. This would also be consistent with the 9:1 ratio of EDOT:PMo $\mathrm{Mo}_{12}$ obtained in the hybrid. When we carried out reaction in air, the reduced $\mathrm{PMo}_{12}$ anion could be easily reoxidized by molecular oxygen, a process that is known to be facile [17]. In this sense, $\mathrm{PMo}_{12}$ would be acting as a catalyst in air, which confirms the ability of $\mathrm{PMo}_{12}$ to oxidize EDOT to PEDOT in the absence of any other reagent. SEM micrographs of the phosphomolybdic acid and of the chemically synthesized

TABLE I Frequency Values $\left(\mathrm{cm}^{-1}\right)$ and Assignment of FTIR Bands Observed for the $\mathrm{H}_{3} \mathrm{PMo}_{12} \mathrm{O}_{40}$ and the PEDOT/PMo 12 Hybrid.

\begin{tabular}{lcc}
\hline Assignment & $\mathrm{H}_{3} \mathrm{PMo}_{12} \mathrm{O}_{40}{ }^{\mathrm{a}}$ & PEDOT/PMo \\
\hline$v_{12} \mathrm{Mo}-\mathrm{O}-\mathrm{Mo}$ (edge sh) & $787(760-800)$ & 805 \\
$v_{2} \mathrm{Mo}-\mathrm{O}-\mathrm{Mo}$ (corner sh) & $869(840-910)$ & 838 \\
$v_{3} \mathrm{Mo}=\mathrm{O}$ (terminal) & $962(960-1000)$ & 957 \\
$v_{4} \mathrm{P}-\mathrm{O}$ & $1064(1060-1080)$ & 1051 \\
\hline
\end{tabular}

${ }^{\mathrm{a}}$ The values within brackets indicate the range of frequencies proposed in the literature [17]. 


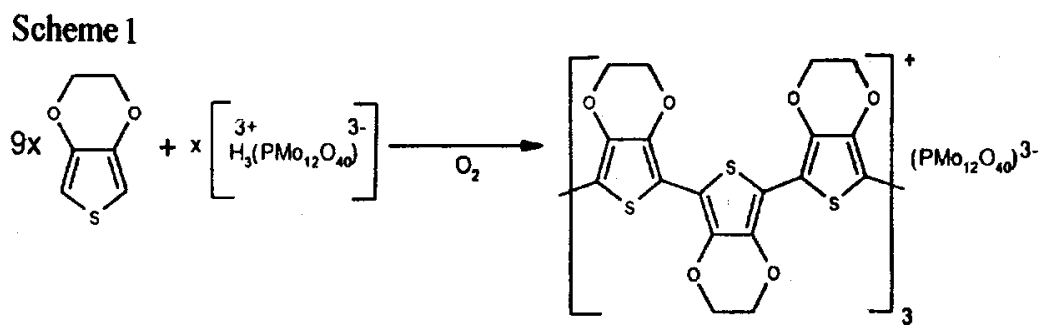

SCHEME 1 Schematic representation of the molecular hybrid material formed between poly(3,4-Ethylene Dioxythiophene and the phosphomolybdate anion.

PEDOT $/ \mathrm{PMo}_{12}$ hybrid are illustrated in Figures 2(a) and 2(b) respectively. It is apparent that the morphological structure of the PEDOT polymer doped with the cluster $\mathrm{PMo}_{12}$ anion, anchored in the polymer matrix, is different from that of the free acid.

The electrical conductivity of the molecular hybrid material of PEDOT/PMo $\mathrm{PM}_{12}$ bulk was measured in the temperature range of $300-400 \mathrm{~K}$ in air. Figures $3(\mathrm{a})$ and $3(\mathrm{~b})$ show the current-voltage $(I-V)$ behavior and the deduced conductivity $(\log \sigma)$ with reciprocal of temperature $(1000 / T)$ respectively. The conductivity is high and increases as a function of

(a)

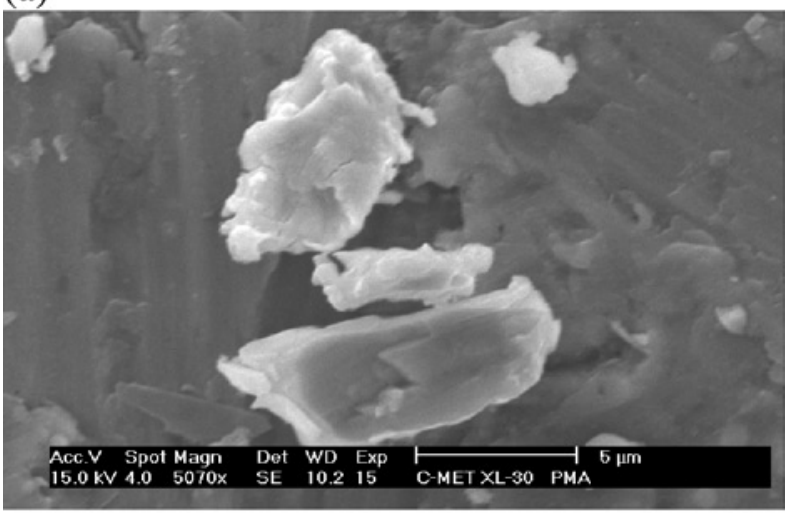

(b)

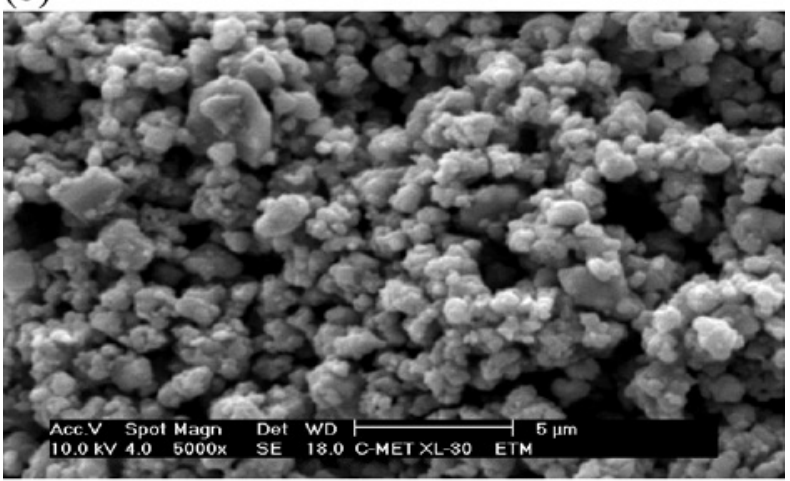

FIGURE 2 SEM micrographs of (a) phosphomolybdic acid and (b) PEDOT/PMo 12 hybrid. 
(a)

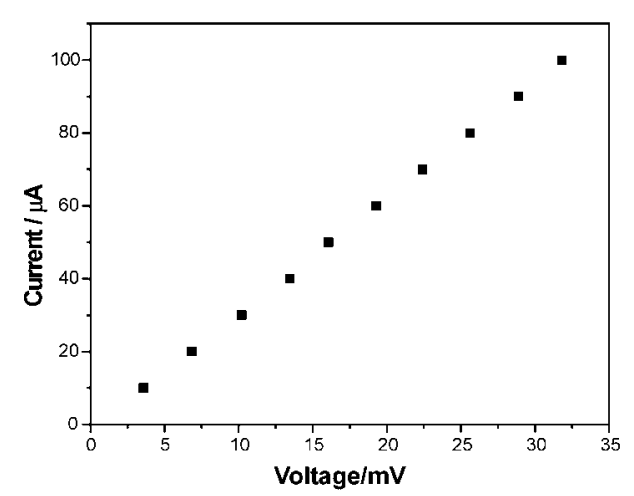

(b)

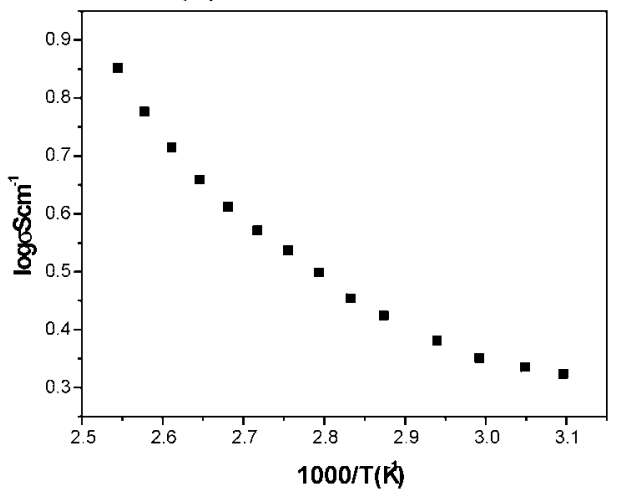

FIGURE 3 Four-probe electrical measurements for PEDOT/PMo ${ }_{12}$ hybrid: (a) current-voltage $(I-V)$ behavior at $300 \mathrm{~K}$ (similar ohmic characteristics are observed at higher temperatures and are not reported here for sake of clarity); (b) deduced evolution $v s$. temperature of the electronic conductivity.

temperature from $\sim 2.0$ to $7.0 \mathrm{~S} \mathrm{~cm}^{-1}$ between 300 and $400 \mathrm{~K}$. It accounts for the expected thermally activated behavior of the hybrid material having $p$-type polaronic conductivity [18].

\section{CONCLUSION}

A new highly conducting organo-inorganic electroactive hybrid material has been synthesized. It consists of a Poly(3,4-Ethylene Dioxythiophene) matrix doped with the active inorganic phosphomolybdate anion. Interesting electrochemical properties for this novel type of hybrid functional material could be expected.

\section{Acknowledgements}

Authors would like to thank Dr. B. K. Das (Executive Director, C-MET) and gratefully acknowledge Messrs Bayer AG Germany, Mr. S. D. Joshi (Bayer India Ltd., Mumbai) and Dr. Tanay Seth for SEM analysis, Mr. R. Marimuthu for thermal analysis, Mr. S. K. Apte for FTIR analysis and Ms. D. Amutha and Mr. K. Thiagarajan, their assistants, regarding the preparation of the manuscript.

\section{References}

[1] Wu, C.-G., DeGroot, D. C., Marcy, H. O., Schindler, J. L., Kannewurf, C. R., Liu, Y.-J., Hirpo, W. and Kanatzidis, M. G. (1996). Chem. Mater, 8, 1992.

[2] Gomez-Romero, P. and Lira-Cantu, M. (1999). J. Solid State Chem., 147, 601.

[3] De Paoli, M.-A., Waltman, R. J., Diaz, A. F. and Bargo, J. (1985). J. Polym. Sc. Polym. Chem. Ed., $23,1687$.

[4] Niwa, O. and Tamamura, T. (1984). J. Chem. Soc. Chem. Commun., 817.

[5] Bidan, G., Genies, E. M. and Lapkowski, M. (1988). J. Chem. Soc. Chem. Commun., 5, 533.

[6] Wang, B. and Dong, S. (1993). Electrochim. Acta., 7, 38.

[7] Lira-Cantu, M. and Gomez-Romero, P. (1998). Chem. Mater, 10, 698.

[8] Otero, T. F., Cheng, S. A. and Huerta, F. (2000). J. Phys. Chem. B., 104, 10522.

[9] Groenendal, L., Jonas, F., Freitage, D., Pielartzik, H. and Reynolds, J. R. (2000). Adv. Mater., $12,481$.

[10] Ghosh, S. and Inganas, O. (1999). Adv. Mater., 11, 1214.

[11] Novak, P., Muller, K., Santhanam, K. S. V. and Haas, O. (1997). Chem. Rev., 97, 207. 
[12] Murugan, A. V., Kale, B. B., Kwon, C.-W., Campet, G. and Vijayamohanan, K. (2001). J. Mater. Chem., 11, 2470.

[13] Murugan, A. V., Kwon, C.-W., Campet, G., Kale, B. B., Maddanimath, T. and Vijayamohanan, K. (2001). J. Power. Sources, 105, 1.

[14] Pope, M. T. (1983). Hetero Poly and Isopoly Oxometalates. Springer, Berlin.

[15] Xi, X. and Dong, S. (1995). Electrochim. Acta., 40, 2785.

[16] Fournier, M., Rocchiccioli-Deltchef, C. and Kazansky, L. P. (1994). Chem. Phys. Lett., $223,297$.

[17] Rao, C. N. (1970). Modern Aspects of Solid State Chemistry. Plenum Press, New York, p. 531.

[18] Mayer (1974). J. Polym. Eng. Sci., 14, 706. 

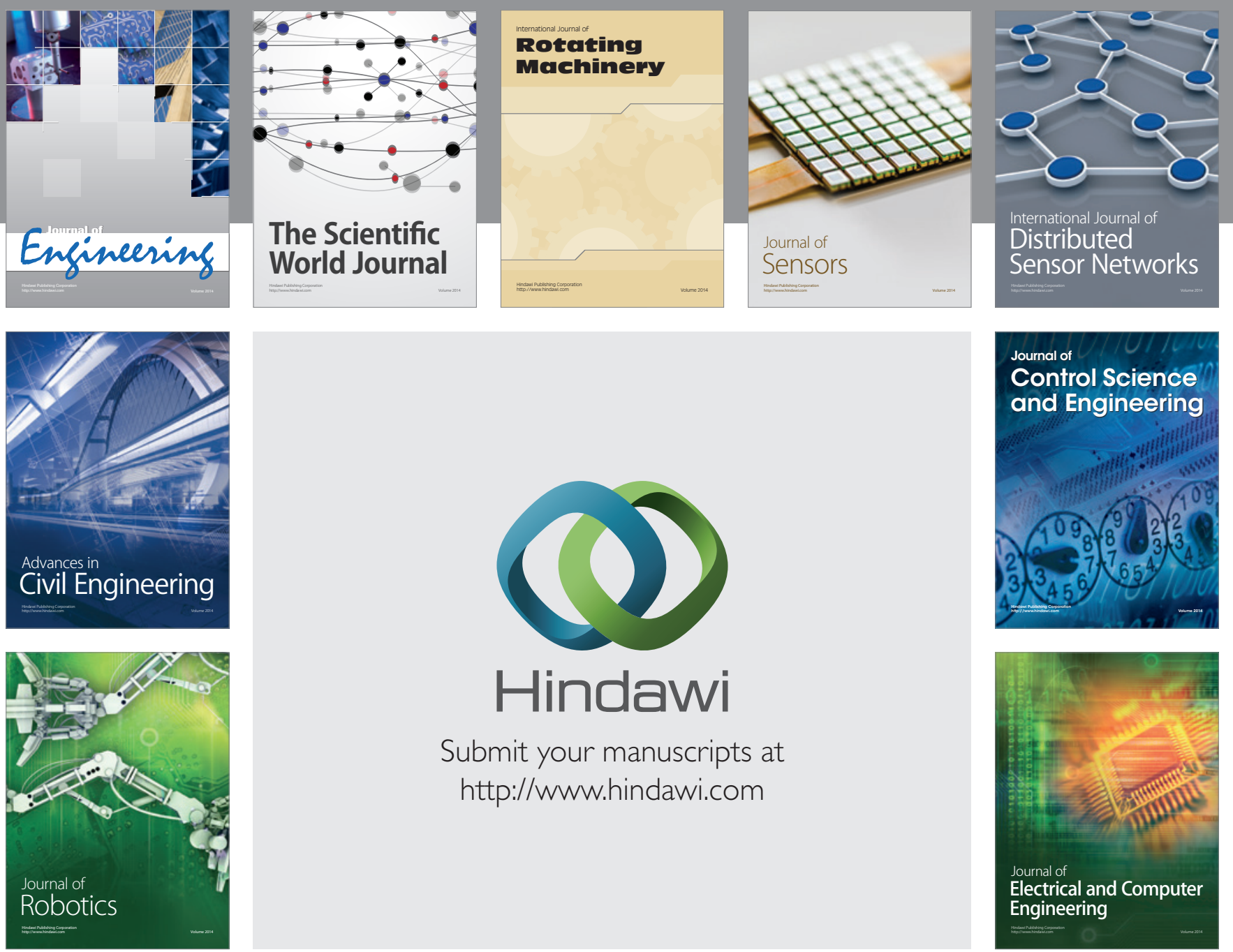

Submit your manuscripts at

http://www.hindawi.com
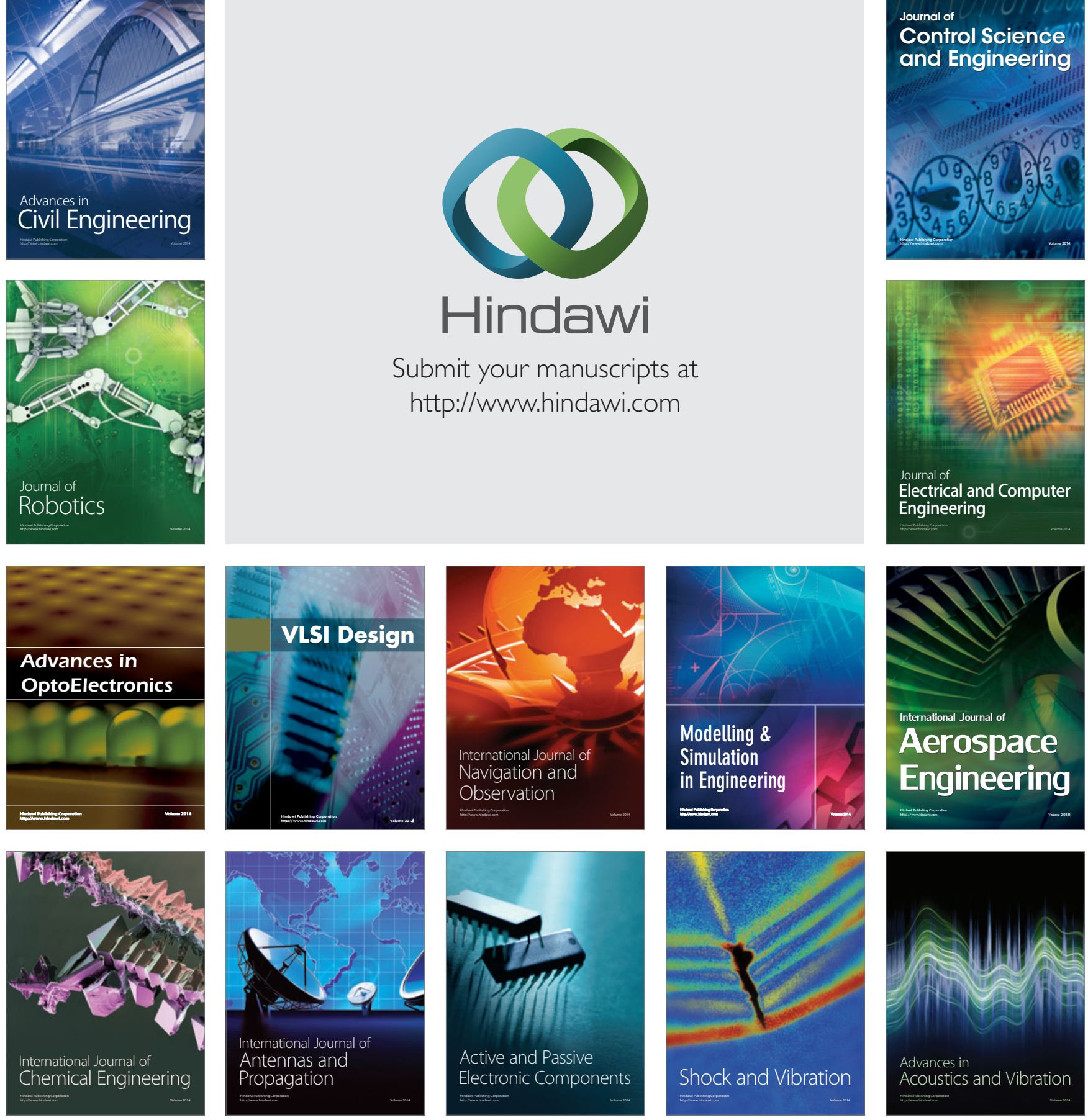